\title{
Impact of biomarkers and primary tumor location on the metastatic colorectal cancer first-line treatment landscape in five European countries
}

\author{
George Kafatos*,1(D), Victoria Banks ${ }^{1}$ (D), Peter Burdon² ${ }^{(\mathbb{D})}$, David Neasham ${ }^{(\mathbb{D})}$, Kimberly A \\ Lowe $^{3 \text { (D) }, ~ C a r o l i n e ~ A n g e r ~}{ }^{4}$, Fil Manuguid ${ }^{4}$ \& Jörg Trojan ${ }^{5}$ (D) \\ ${ }^{1}$ Center for Observational Research, Amgen Ltd., 1 Uxbridge Business Park, Sanderson Road, Uxbridge, UB8 1DK, UK \\ ${ }^{2}$ Medical Affairs, Amgen (Europe) GmbH, Suurstoffi 22, Postfach 94, Rotkreuz 6343, Switzerland \\ ${ }^{3}$ Center for Observational Research, Amgen Inc., One Amgen Center Drive, MS D2262, Thousand Oaks, CA 91320, USA \\ ${ }^{4}$ IQVIA Real-World \& Analytics solutions, 210 Pentonville Road, London, N1 9JY, UK \\ ${ }^{5}$ Medizinische Klinik 1, Universitätsklinikum Frankfurt, Goethe-Universität, Theodor-Stern-Kai 7, Frankfurt am Main, 60590, \\ Germany \\ *Author for correspondence: Tel.: +44 189552 5615; gkafatos@amgen.com
}

Background: Advances in therapies for patients with metastatic colorectal cancer (mCRC) and improved understanding of prognostic and predictive factors have impacted treatment decisions. Materials \& methods: This study used a large oncology database to investigate patterns of monoclonal antibody (mAb) plus chemotherapy treatment in France, Germany, Italy, Spain and the UK in mCRC patients treated in first line in 2018. Results: Anti-EGFR mAbs were most often administered to patients with RAS wild-type mCRC and those with left-sided tumors, while anti-VEGF mAbs were preferred in RAS mutant and right-sided tumors. Adopted treatment strategies differed between countries, largely due to reimbursement. Conclusion: Biomarker status and primary tumor location steered treatment decisions in first line. Adopted treatment strategies differed between participating countries.

Lay abstract: Each patient's cancer is unique. For example, colon cancer on the left side is different from colon cancer on the right side. Colon cancer is different from cancer of the rectum. Cancers also have changes in their genes, which means some treatments should work, while others may not. Doctors can select among different medicines to find the drug that works best for each patient. We looked at patients with cancer of the colon or rectum that has spread to other organs. We tried to find out how doctors in Europe select drugs for their patients after performing tests called RAS or BRAF. We found that doctors make different choices in different countries.

First draft submitted: 23 September 2020; Accepted for publication: 11 December 2020; Published online: 19 January 2021

Keywords: $B R A F \bullet \mathrm{EGFR} \bullet \mathrm{mCRC} \bullet$ primary tumor location $\bullet$ RAS $\bullet$ tumor sidedness $\bullet$ VEGF

During the last decade, improvements in the treatment of metastatic colorectal cancer (mCRC) have increased median survival time for patients from 12 months to approximately 3 years [1,2]. Major drivers of this success were the development of an antiangiogenic agent, the VEGF inhibitor bevacizumab, of monoclonal antibodies (mAbs) that inhibit the EGFR namely panitumumab and cetuximab, and a better understanding of prognostic and predictive biomarkers and of the different molecular profiles of left- and right-sided tumors $[3,4,5]$. Further important factors were the improvements in the adoption of multi-disciplinary teams/tumor boards, new surgical techniques, or local ablative therapies [6].

Chemotherapy, anti-VEGF mAbs and anti-EGFR mAbs are now the mainstay of systemic mCRC therapy. In the first line following diagnosis of $\mathrm{mCRC}$, treatment is planned according to patient fitness, resectability of the tumor and/or metastases, and the tumor's biomarker status [7]. The European Society for Medical Oncology (ESMO) recommends the use of biologicals (targeted agents) as first line of treatment for most patients unless 
contraindicated [7]. According to EMA UK label, the anti-VEGF mAb bevacizumab should be used in combination with fluoropyrimidine-based chemotherapy [8]. Anti-EGFR mAbs should be used in combination with FOLFOX or FOLFIRI or as monotherapy in patients who have failed oxaliplatin- or irinotecan-based therapy, are intolerant of irinotecan (cetuximab) or have failed fluoropyrimidine-based therapy (panitumumab). Both are limited to patients with $R A S$ wild-type tumors $[9,10]$. There is strong evidence that $B R A F$ mutation is predictive for a lack of benefit from anti-EGFR mAbs, although some discussions remain [7,11]. Evidence from the BEACON trial suggests some benefit of adding anti-EGFR mAb-based therapy to $B R A F / M E K$ inhibitors. Anti-EGFR mAbs are thought to block the anticipated escape mechanism resulting from $B R A F / M E K$ inhibition [12].

Primary tumor location, a surrogate of the different molecular profiles of left- and right-sided tumors, although first described in 2001 [13], has gained increasing attention after a 2017 meta-analysis of its prognostic and predictive value in patients with $R A S$ wild-type mCRC [14]. In this retrospective analysis, six randomized trials (CRYSTAL, FIRE-3, CALGB 80405, PRIME, PEAK and 20050181) were pooled, comparing chemotherapy plus anti-EGFR mAb therapy with chemotherapy or chemotherapy plus bevacizumab. A worse prognosis for overall survival, progression-free survival and objective response rate (ORR) was found for patients with right-sided primary tumors. Tumor side was also found to be predictive of treatment efficacy, with the greatest effect in patients with left-sided tumors receiving anti-EGFR mAb in combination with chemotherapy. Similar results were found when analyzing the anti-EGFR mAbs separately in the panitumumab trials PRIME $[4,15,16]$ and PEAK $[4,16,17]$ and the cetuximab trials CRYSTAL and FIRE-3 [18].

The present study aimed to capture the treatment patterns in the first line of therapy of mCRC patients actively treated in 2018 in real-world clinical practice in five European countries by tumor sidedness and biomarker status.

\section{Materials \& methods}

\section{Database}

This was a retrospective analysis using a large oncology database (Oncology Dynamics ${ }^{\top M}$, IQVIA Ltd., London, the UK). The database was designed as a cross-sectional physician survey that collects anonymized individual-level information on drug-treated cancer patients in Europe (and other non-European countries) regardless of cancer type, disease stage and/or treatment $[19,20,21,22,23,24,25,26]$. The database has been described in detail elsewhere [27].

In brief, the database includes data from oncology centers in 10 countries (France, Germany, Italy, Spain and the UK, China, Japan, South Korea, Saudi Arabia and Mexico). It is designed as repeated quarterly crosssectional cohorts and contains more than 167,000 cancer cases per year and over 35 cancer indications. The database captures patient information via a standardized electronic case report form entered by the treating physician from patients' health records. Stratified random sampling is used to select physicians to represent the distribution of specialties for each cancer indication and country. It is limited to patients treated with a cancer drug at the time of data collection and excludes patients solely treated with radiotherapy, surgery, supportive care or on active surveillance.

\section{Objectives}

The study objective was to describe the demographic and clinical characteristics, as well as treatment patterns, i.e. anti-EGFR $m A b s$, anti-VEGF $m A b s$ and/or chemotherapy, for patients treated for $\mathrm{mCRC}$ in first line during 2018 by tumor sidedness and biomarker status.

\section{Eligibility criteria}

All mCRC patients from the participating countries recorded in the database who received active anti-cancer first-line treatment in the advanced/metastatic setting in 2018, fulfilled the International Classification of Diseases, Tenth Revision codes used to define the mCRC population, and had a diagnosis date between July 2013 and December 2018, were included. Patients with unknown $R A S$ or $B R A F$ status, or unknown primary tumor location were excluded. Clinical trial participants were excluded.

\section{Demographic \& clinical characteristics}

Description of demographics included country, age, sex, treatment facility site type and subtype. Clinical characteristics included the quarter of diagnosis, the BMI, Eastern Cooperative Oncology Group performance status, stage at diagnosis, site of metastasis and location of primary tumor. 


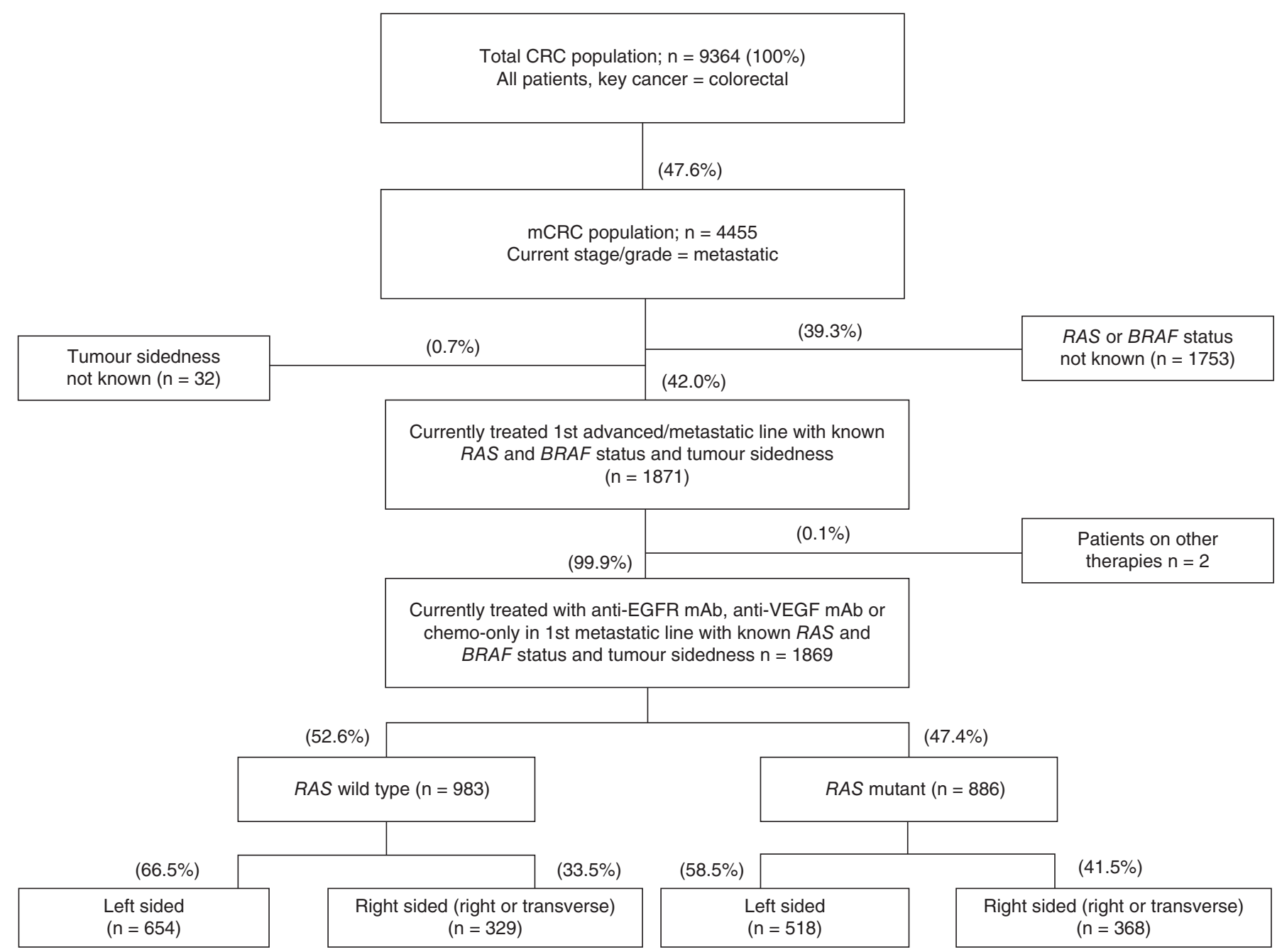

Figure 1. CONSORT flow diagram describing the primary tumor location and biomarker status of first line of therapy for metastatic colorectal cancer patients.

CONSORT: Consolidated standards of reporting trials; CRC: Colorectal cancer; mCRC: Metastatic colorectal cancer.

Left-sided tumors were defined as those originating in the splenic flexure, descending colon, sigmoid colon or rectum. Right-sided tumors were defined as those originating in the appendix, cecum, ascending colon or hepatic flexure as well as the transversum - between the hepatic and splenic flexure; this is in line with previous analyses, such as the meta-analysis of six trials mentioned above [14]. Tumor sidedness was determined by the treating physician. Where tumor sidedness was recorded as unknown by the physician, the International Classification of Diseases, Tenth Revision code, from which anatomy of tumor location could be determined, was used where available.

\section{Statistical considerations}

Frequencies and proportions were provided for mCRC patients within the selected cohorts with their corresponding 95\% Binomial Exact CIs. SAS Software was used (SAS Enterprise Guide 7.1.; SAS Institute Inc., NC, USA).

\section{Results}

\section{Patient \& tumor characteristics}

There were $4455 \mathrm{mCRC}$ patients within the database who were actively drug treated in 2018. A full description of all 4455 patients can be found elsewhere [27]. Of these, $42.0 \%(\mathrm{n}=1871)$ were receiving their first advanced/metastatic line of therapy and thus eligible for the present analysis; their $R A S$ and $B R A F$ status as well as their primary tumor location were known. Of these 1871 patients, two were excluded from the analysis as they received therapy other than anti-EGFR mAbs plus chemotherapy, anti-VEGF mAbs plus chemotherapy or only chemotherapy (see 
CONSORT [Consolidated Standards of Reporting Trials] flow diagram in Figure 1); 635 patients were from Italy, 337 from Germany, 318 each from France and the UK and 261 from Spain. The oncology center characteristics for the 1869 analyzed patients are provided in the supplemental material (Supplementary Table 1). Of patients, $60.8 \%(\mathrm{n}=1136)$ were male, $68.6 \%(\mathrm{n}=1282)$ were older than 60 years of age. Overall, $52.6 \%(\mathrm{n}=983)$ had $R A S$ wild-type tumors and $6.5 \%(\mathrm{n}=122)$ had BRAF mutant tumors; $62.7 \%(\mathrm{n}=1172)$ had left-sided and $37.3 \%$ ( $\mathrm{n}=697$ ) had right-sided or transverse primary tumors (summarized as right-sided). Left-sided tumors were $R A S$ wild type in $55.8 \%$ of patients $(\mathrm{n}=654)$. Of right-sided tumors, slightly fewer than half, $47.2 \%(\mathrm{n}=329)$, were $R A S$ wild type. BRAF wild type status was found in $95.7 \%(\mathrm{n}=1122)$ of left-sided and $89.7 \%(\mathrm{n}=625)$ of right-sided tumors (Table 1). Further patient and tumor characteristics are listed in Table 1.

\section{Treatment landscape}

Patients with $R A S$ wild-type tumors were most commonly treated with anti-EGFR mAbs plus chemotherapy (62.6\%; 95\% CI: 59.5\%, 65.6\%). The remaining patients were treated equally with anti-VEGF mAbs plus chemotherapy (18.4\%; 95\% CI: 16.0\%, 21.0\%) or chemotherapy-only (19.0\%; 95\% CI: 16.6\%, 21.6\%). Patients with $R A S$ mutant tumors were most commonly prescribed anti-VEGF mAbs plus chemotherapy $(60.5 \%$; $95 \% \mathrm{CI}$ : $57.2 \%, 63.7 \%$ ) followed by chemotherapy-only (38.1\%; 95\% CI: 34.9\%, 41.4\%). There were 12 patients (out of 886 RAS mutant patients) documented as receiving anti-EGFR mAbs plus chemotherapy (1.4\%; $95 \%$ CI: $0.7 \%$, 2.4\%; Table 2).

Of the $983 R A S$ wild-type patients, 654 (66.5\%) had left-sided tumors and 329 (33.4\%) had right-sided tumors. For both groups of patients, the most common treatment was anti-EGFR mAbs plus chemotherapy $(71.6 \%$; $95 \%$ CI: $67.9 \%, 75.0 \%$ and $44.7 \%$; 95\% CI: 39.2\%, 50.2\%) for left- and right-sided tumors, respectively). Of the 886 RAS mutant patients, 518 (58.4\%) had left-sided tumors and 368 (41.5\%) had right-sided tumors. For both leftand right-sided RAS mutant tumors, the majority were treated with anti-VEGF mAbs plus chemotherapy (59.5\%; 95\% CI: 55.1\%, 63.7\%) for left-sided and 62.0\%; 95\% CI: 56.8\%, 66.9\% for right-sided) (Table 2).

Of the RAS wild-type patients, $108(11.0 \%)$ were BRAF mutant. For tumors of both $R A S$ and $B R A F$ wild type status, the most common treatment was anti-EGFR mAbs plus chemotherapy (69.3\%; 95\% CI: 66.1\%, 72.4\%). For tumors of $R A S$ wild type and $B R A F$ mutant status the most common treatment were anti-VEGF mAbs plus chemotherapy (44.4\%; 95\% CI: 34.9\%, 54.3\%). Of the RAS mutant patients, 872 (98.4\%) were $B R A F$ wild type and $14(1.6 \%)$ were documented as $B R A F$ mutant. For $R A S$ mutant and $B R A F$ wild-type tumors the most common treatment were anti-VEGF mAbs (61.1\%; 95\% CI: 57.8\%, 64.4\%). In the rare case of patients who were reported as having both $R A S$ and $B R A F$ mutant tumor status $(\mathrm{n}=14)$ the great majority was treated with chemotherapy only (78.6\%; 95\% CI: 49.2\%, 95.3\%) (Table 3).

When looking at the treatment landscape by country, there were differences regarding the adopted treatment strategies. In RAS wild-type mCRC patients, anti-VEGF mAbs plus chemotherapy were not prescribed in the UK as opposed to the other countries, in which anti-VEGF mAb-based combinations ranged from $15.2 \%$ in Spain to $25.6 \%$ in Italy. Patients with RAS mutant tumors also received anti-VEGF mAbs plus chemotherapy less frequently in the UK $(5.1 \%$; $95 \%$ CI: $2.2 \%, 9.8 \%)$ compared with other countries which ranged from $66.9 \%$ in France to $78.9 \%$ in Italy (Supplementary Tables $2 \& 3$ ).

\section{Discussion}

This study aimed to capture the treatment patterns in mCRC patients in real-world clinical practice in five European countries. There were large differences in treatment patterns by biomarker status and primary tumor location. RAS wild-type patients were mainly treated with anti-EGFR mAbs plus chemotherapy (62.6\%; 95\% CI: 59.5\%, 65.6\%) whereas RAS mutant patients were most commonly treated with anti-VEGF mAbs plus chemotherapy (60.5\%; 95\% CI: $57.2 \%, 63.7 \%)$. For $R A S$ wild-type patients, anti-EGFR mAbs plus chemotherapy were more frequently prescribed in patients with left-sided compared with right-sided tumors (71.6\%; 95\% CI: $67.9 \%, 75.0 \%$ vs 44.7\%; 95\% CI: 39.2\%, 50.2\%, respectively). In $R A S$ and $B R A F$ wild-type patients, the most common treatment was anti-EGFR mAb plus chemotherapy (69.3\%; 95\% CI: 66.1\%, 72.4\%). RAS wild-type/BRAF mutant patients preferably received anti-VEGF mAbs (44.4\%; 95\% CI: 34.9\%, 54.3\%). For $R A S$ mutant/ $B R A F$ wild-type patients, anti-VEGF mAbs plus chemotherapy was most commonly prescribed (61.1\%; 95\% CI: 57.8\%, 64.4\%).

Studies with a broad focus evaluating the patterns of mCRC treatment, especially in first line, are rare; the authors are not aware of any studies using a similarly broad research angle as theirs. The predecessor of the database that was used in the present study (Oncology Analyzer ${ }^{\top M}$ ), was previously used to evaluate treatment patterns in 
Table 1. Patient demographics and disease characteristics.

Parameters, $\mathbf{n}(\%)$

Sex

- Female

- Male

Age group at current line of therapy

$-<16$

$-16-50$

$-51-60$

$-61-75$

$-\geq 76$

ECOG status at current line of therapy

$\begin{array}{ll}-0 & 390(33.3)\end{array}$

$-1$

$-2$

$-3$

$-4$

- Unknown

Stage at diagnosis

- Stage I

- Stage II

- Stage III

- Stage IV

- Unknown

Primary tumor location

- Left-sided

- Right-sided

- Transverse

RAS status

- Wild type

- Mutant

BRAF status

- Wild type

- Mutant

Site of metastasis

- Liver \& lung combination

- Liver only

- Liver with other combination

- Lung only

- Lung with other combination

- Other

Pre-existing comorbidities

- No

- Yes

Type of comorbidities

- Auto-immune disease

- Bone disease

- Cardiovascular

- Gastrointestinal

${ }^{\dagger}$ Right-sided tumors include tumors of the transverse colon.

CRC: Colorectal cancer; ECOG: Eastern cooperative oncology group; NA: Not available.
Left-sided tumors

$(n=1172)$

$445(38.0)$

$727(62.0)$

$102(8.7)$

$286(24.4)$

$591(50.4)$

193 (16.5)

649 (55.4)

$120(10.2)$

$3(0.3)$

1 (0.1)

$9(0.8)$

$10(0.9)$

61 (5.2)

156 (13.3)

905 (77.2)

40 (3.4)

$1172(100.0)$

$-$

$-$

654 (55.8)

518 (44.2)

$1122(95.7)$

50 (4.3)

281 (24.0)

343 (29.3)

303 (25.9)

63 (5.4)

$59(5.0)$

123 (10.5)

541 (46.2)

631 (53.8)

15 (1.3)

12 (1.0)

215 (18.3)

29 (2.5)
Right-sided tumors ${ }^{\dagger}$

( $n=697$ )

288 (41.3)

409 (58.7)

$73(10.5)$

126 (18.1)

355 (50.9)

143 (20.5)

219 (31.4)

408 (58.5)

60 (8.6)

$6(0.9)$

$1(0.1)$

$3(0.4)$

4 (0.6)

29 (4.2)

84 (12.1)

558 (80.1)

22 (3.2)

$-$

593 (85.1)

104 (14.9)

329 (47.2)

368 (52.8)

625 (89.7)

72 (10.3)

164 (23.5)

193 (27.7)

171 (24.5)

31 (4.4)

26 (3.7)

$112(16.1)$

334 (47.9)

363 (52.1)

4 (0.6)

10 (1.4)

119 (17.1)

12 (1.7)
Overall

( $n=1869)$

733 (39.2)

$1136(60.8)$

-

175 (9.4)

412 (22.0)

946 (50.6)

336 (18.0)

609 (32.6)

1057 (56.6)

180 (9.6)

$9(0.5)$

$2(0.1)$

$12(0.6)$

$14(0.7)$

90 (4.8)

240 (12.8)

1463 (78.3)

62 (3.3)

$1172(62.7)$

593 (31.7)

$104(5.6)$

983 (52.6)

886 (47.4)

1747 (93.5)

122 (6.5)

445 (23.8)

536 (28.7)

474 (25.4)

94 (5.0)

85 (4.5)

235 (12.6)

875 (46.8)

994 (53.2)

19 (1.0)

$22(1.2)$

334 (17.9)

$41(2.2)$ 
Table 1. Patient demographics and disease characteristics (cont.).

\begin{tabular}{|llll|}
\hline Parameters, $\mathbf{n}(\%)$ & $\begin{array}{l}\text { Left-sided tumors } \\
(\mathbf{n = 1 1 7 2 )}\end{array}$ & $\begin{array}{l}\text { Right-sided tumors } \\
(\mathbf{n}=697)\end{array}$ & $\begin{array}{l}\text { Overall } \\
(\mathbf{n}=1869)\end{array}$ \\
\hline - Infection & $5(0.4)$ & $1(0.1)$ & $6(0.3)$ \\
\hline - Metabolic & $224(19.1)$ & $134(19.2)$ & $358(19.2)$ \\
\hline - Neurological & $36(3.1)$ & $21(3.0)$ & $57(3.0)$ \\
\hline - Renal & $41(3.5)$ & $34(4.9)$ & $75(4.0)$ \\
\hline - Respiratory & $155(13.2)$ & $97(13.9)$ & $252(13.5)$ \\
\hline - Other & $223(19.0)$ & $114(16.4)$ & $337(18.0)$ \\
\hline - None & $513(43.8)$ & $309(44.3)$ & $822(44.0)$ \\
\hline - NA & $28(2.4)$ & $25(3.6)$ & $53(2.8)$ \\
\hline CRC-related surgery & & & \\
\hline - No surgery & $548(46.8)$ & $308(44.2)$ & $856(45.8)$ \\
\hline - Surgery & $624(53.2)$ & $389(55.8)$ & $1013(54.2)$ \\
\hline
\end{tabular}

$\dagger$ Right-sided tumors include tumors of the transverse colon.

CRC: Colorectal cancer; ECOG: Eastern cooperative oncology group; NA: Not available.

\begin{tabular}{|c|c|c|c|c|c|c|}
\hline \multirow[t]{2}{*}{ Treatment } & \multicolumn{3}{|c|}{ RAS wild type } & \multicolumn{3}{|c|}{ RAS mutant } \\
\hline & Left-sided & Right-sided & Total & Left-sided & Right-sided & Total \\
\hline Anti-VEGF mAbs & $\begin{array}{l}76(11.6) \\
{[9.3,14.3]}\end{array}$ & $\begin{array}{l}105(31.9) \\
{[26.9,37.3]}\end{array}$ & $\begin{array}{l}181(18.4) \\
{[16.0,21.0]}\end{array}$ & $\begin{array}{l}308(59.5) \\
{[55.1,63.7]}\end{array}$ & $\begin{array}{l}228(62.0) \\
{[56.8,66.9]}\end{array}$ & $\begin{array}{l}536(60.5) \\
{[57.2,63.7]}\end{array}$ \\
\hline Total & $\begin{array}{l}654(100.0) \\
{[99.4,100.0]}\end{array}$ & $\begin{array}{l}329(100.0) \\
{[98.9,100.0]}\end{array}$ & $\begin{array}{l}983(100.0) \\
{[99.6,100.0]}\end{array}$ & $\begin{array}{l}518(100.0) \\
{[99.3,100.0]}\end{array}$ & $\begin{array}{l}368(100.0) \\
{[99.0,100.0]}\end{array}$ & $\begin{array}{l}886(100.0) \\
{[99.6,100.0]}\end{array}$ \\
\hline
\end{tabular}

Chemo: Chemotherapy; mAbs: Monoclonal antibodies.

\begin{tabular}{|c|c|c|c|c|c|c|}
\hline \multirow[t]{2}{*}{ Treatment } & \multicolumn{3}{|c|}{ RAS wild type } & \multicolumn{3}{|c|}{ RAS mutant } \\
\hline & $B R A F$ wild type & $B R A F$ mutant & Total & $B R A F$ wild type & BRAF mutant & Total \\
\hline Anti-EGFR mAbs & $\begin{array}{l}589(69.3) \\
{[66.1,72.4]}\end{array}$ & $\begin{array}{l}26(24.1) \\
{[16.4,33.3]}\end{array}$ & $\begin{array}{l}615(62.6) \\
{[59.5,65.6]}\end{array}$ & $\begin{array}{l}12(1.4) \\
{[0.7,2.4]}\end{array}$ & $\begin{array}{l}0(0.0) \\
{[0.0,23.2]}\end{array}$ & $\begin{array}{l}12(1.4) \\
{[0.7,2.4]}\end{array}$ \\
\hline Anti-VEGF mAbs & $\begin{array}{l}133(15.6) \\
{[13.3,18.3]}\end{array}$ & $\begin{array}{l}48(44.4) \\
{[34.9,54.3]}\end{array}$ & $\begin{array}{l}181(18.4) \\
{[16.0,21.0]}\end{array}$ & $\begin{array}{l}533(61.1) \\
{[57.8,64.4]}\end{array}$ & $\begin{array}{l}3(21.4) \\
{[4.7,50.8]}\end{array}$ & $\begin{array}{l}536(60.5) \\
{[57.2,63.7]}\end{array}$ \\
\hline Chemo only & $\begin{array}{l}153(17.5) \\
{[15.0,20.2]}\end{array}$ & $\begin{array}{l}34(31.5) \\
{[22.9,41.1]}\end{array}$ & $\begin{array}{l}187(19.0) \\
{[16.6,21.6]}\end{array}$ & $\begin{array}{l}327(37.5) \\
{[34.3,40.8]}\end{array}$ & $\begin{array}{l}11(78.6) \\
{[49.2,95.3]}\end{array}$ & $\begin{array}{l}338(38.1) \\
{[34.9,41.4]}\end{array}$ \\
\hline Total & $\begin{array}{l}875(100.0) \\
{[99.6,100.0]}\end{array}$ & $\begin{array}{l}108(100) \\
{[96.6,100.0]}\end{array}$ & $\begin{array}{l}983(100.0) \\
{[99.6,100.0]}\end{array}$ & $\begin{array}{l}872(100.0) \\
{[99.6,100.0]}\end{array}$ & $\begin{array}{l}14(100.0) \\
{[76.8,100.0]}\end{array}$ & $\begin{array}{l}886(100.0) \\
{[99.6,100.0]}\end{array}$ \\
\hline
\end{tabular}

Chemo: Chemotherapy; mAbs: Monoclonal antibodies.

the USA, the EU (France, Germany, Italy, Spain), the UK and Japan using data from 2007 [21], and in France, Germany, Italy and Spain using a 2009 data snapshot [28]. In 2007, treatment combinations including targeted therapy (bevacizumab) accounted for a limited proportion of administered regimens in the first-line mCRC setting, with the highest uptake in France with $<20 \%$, followed by Italy and Germany with $<10 \%$ [21]. By 2009, the proportion of bevacizumab-containing regimens had increased to approximately $40 \%$ in France, Germany and Italy, and 30\% in Spain; cetuximab-containing regimens accounted for 7-14\% [28]. Approved indications have changed substantially since these studies were conducted, generally allowing for refined use of these agents. The anti-EGFR $\mathrm{mAbs}$ labels changed several times over the last years, including changes in mandatory wild type status of $K R A S$ to $R A S$ and of allowed chemotherapy backbone (Figure 2). Panitumumab was initially approved as monotherapy, then in combination with FOLFOX in first line and with FOLFIRI in second-line; the combination with FOLFIRI 


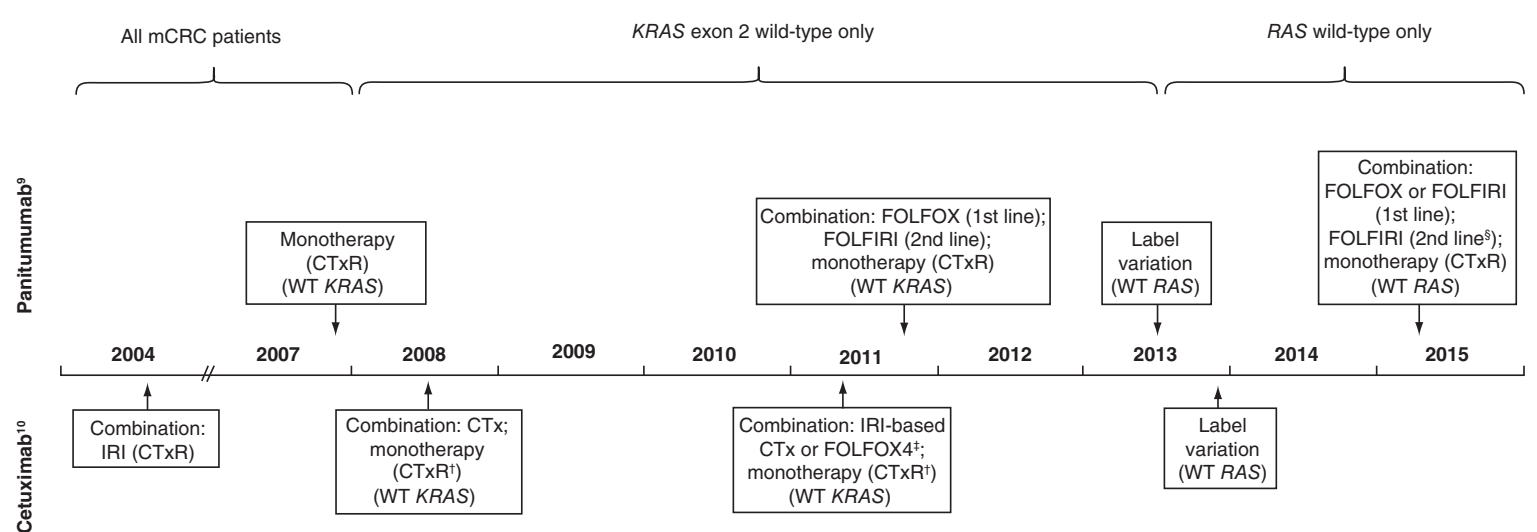

Figure 2. Selected European union label changes relating to the patient population eligible for anti-EGFR monoclonal antibody therapy.

${ }^{\dagger}$ And intolerant to irinotecan.

¥FOLFOX4 subsequently revised to FOLFOX (first line) in 2012.

$\S$ For patients who have received first-line fluoropyrimidine-based chemotherapy (excluding irinotecan).

Therapeutic indications have been abbreviated; please see [9] (panitumumab) and [10] (cetuximab) for full details.

CTx: Chemotherapy; CTxR: Chemotherapy refractory; FOLFOX: Folinic acid, fluorouracil and oxaliplatin; FOLFIRI:

Folinic acid, fluorouracil and irinotecan; IRI: Irinotecan; mAb: Monoclonal antibody; WT: Wild type.

in first line followed in 2015 [29]. Additionally, ESMO guidelines were continuously updated to incorporate new clinical evidence [7] and entailed considerable shifts in treatment practices.

The meta-analysis of six Phase III trials by Arnold et al. provided strong evidence about the prognostic and predictive value of primary tumor location, which are biologically different [5]. It was shown that the greatest effect was obtained in patients with left-sided tumors receiving anti-EGFR mAbs in combination with chemotherapy [14]. In the present study, $71.6 \%$ (95\% CI: 67.9\%, 75.0\%) of patients with left-sided $R A S$ wild-type tumors and $44.7 \%$ (95\% CI: 39.2\%, 50.2\%) of patients with right-sided $R A S$ wild-type tumors received anti-EGFR mAbbased therapy, using a chemotherapy backbone. In the UK, the great majority of $R A S$ mutant patients were treated with chemotherapy only (93.6\%; 95\% CI: 88.6\%, 96.9\%) whereas in the other European countries most patients were treated with anti-VEGF mAbs plus chemotherapy (between 67.2 and $78.9 \%$ in Germany and Italy, respectively). In the UK, National Institute for Health and Care Excellence (NICE) does not recommend the anti-VEGF $m A b$ bevacizumab in combination with FOLFOX or oxaliplatin plus capecitabine (CAPEOX) for the treatment of $\mathrm{mCRC}$ [32] and it was removed from the UK cancer drug fund in November 2016 [33]. Apart from the differences in reimbursement in the different countries, it needs to be highlighted that the scientific evidence supporting a benefit of bevacizumab in patients with $R A S$ mutant tumors is yet unclear, as was shown by a recent systematic review and network meta-analysis of ten papers reporting six randomized-controlled trials [34]. This analysis found a statistically nonsignificant benefit in progression-free survival and no benefit in overall survival for patients with $R A S$ mutant $\mathrm{mCRC}$ receiving bevacizumab plus chemotherapy versus those receiving chemotherapy alone [34]. Prospective randomized, controlled clinical trials enrolling solely $R A S$ mutant patients have not been conducted with bevacizumab. Interestingly, $1.4 \%$ (95\% CI: 0.7\%, 2.4\%) of patients with $R A S$ mutant tumors were documented to have received anti-EGFR mAbs plus chemotherapy. Patients with $R A S$ mutant or unknown status should not receive anti-EGFR mAbs and it thus seems likely that this number is an error in either documentation or treatment choice. The tumors of 14 patients were documented as having both, a $R A S$ and a BRAF mutation. $R A S$ and $B R A F$ mutations are considered as mutually exclusive although some rare cases have been reported in the literature $[35,36,37]$ and there is evidence that with next-generation sequencing (NGS) more such cases might be identified $[38,39]$. Clinical implications of such concomitant mutations are still undetermined.

The topic of primary tumor location as a surrogate for biologically different tumor entities was accepted on a broader scale after the publication of the large meta-analysis of six clinical trials conducted by Arnold et al. [14]. From that time onwards, extensive research was conducted to investigate the prognostic potential of primary tumor location on outcomes, although much of this research was retrospective. Although the 2016 ESMO recommendations could not yet take into consideration the primary tumor location as they predated the metaanalysis, the ESMO pan-Asian guidelines have been updated to include treatment recommendations including 
consideration of left- versus right-sided primary tumor location [40]. The pan-Asian ESMO guidelines recommend that for patients with left-sided $R A S$ wild-type disease, a cytotoxic doublet such as FOLFOX or FOLFIRI plus an anti-EGFR mAb should be the treatment of choice, whereas for those with right-sided $R A S$ wild-type tumors, the cytotoxic triplet FOLFOXIRI plus bevacizumab should be or a cytotoxic doublet plus an anti-EGFR mAb can be, the treatment of choice [40].

There are some limitations to the present analysis. This study focused on describing the treatment patterns by biomarker status and tumor sidedness. However, there was less focus on the impact on clinical characteristics, such as resectability of the metastases or patient fitness of the choice of treatment. The database does not capture data on hospitalizations or survival. Observed country differences might be partly explained by differences in local treatment guidelines, physician prescribing behaviors, or reimbursement policies, some of which have been outlined above but no systematic analysis of the impact of these factors on treatment choice was conducted. A discussion of the database itself can be found elsewhere [27] .

\section{Conclusion}

In clinical practice in the five participating European countries, $R A S$ wild-type patients were mainly treated with anti-EGFR mAbs plus chemotherapy whereas $R A S$ mutant patients were most commonly treated with antiVEGF mAbs plus chemotherapy in all countries except for the UK, where they were more commonly treated with chemotherapy-only in the UK. In $R A S$ wild-type patients, the presence of a $B R A F$ mutation diversified the prescribed treatments compared with $B R A F$ wild-type patients.

\section{Future perspective}

In the era of precision medicine, there are many biomarker-driven drugs that are under development, especially within the oncology therapeutic area. As these drugs are gradually being introduced, it is important to understand the impact of biomarker status in the physicians' treatment decision making.

\section{Summary points}

- Advances in therapies for patients with metastatic colorectal cancer (mCRC) and improved understanding of prognostic and predictive factors such as biomarkers or the recognition of the clinical impact of the biological differences between left- and right-sided primary tumors have impacted mCRC treatment patterns.

- The present study used a large international oncology database to investigate the treatment landscape of anti-VEGF monoclonal antibodys (mAbs) and anti-EGFR mAbs, both in combination with chemotherapy or chemotherapy alone in the first line of therapy of $\mathrm{mCRC}$ patients actively treated in 2018 in real-world clinical practice in five European countries (France, Germany, Italy, Spain and the UK) by tumor sidedness and biomarker status.

- Of the 1869 analyzed patients, RAS wild-type patients were mainly treated with anti-EGFR mAbs plus chemotherapy $(62.6 \% ; 95 \% \mathrm{Cl}: 59.5 \%, 65.6 \%)$ whereas RAS mutant patients were most commonly treated with anti-VEGF mAbs plus chemotherapy $(60.5 \% ; 95 \% \mathrm{Cl}: 57.2 \%, 63.7 \%)$.

- Of RAS wild-type patients with left-sided primary tumors, $71.6 \%$ (95\% Cl: $67.9 \%, 75.0 \%)$ received anti-EGFR mAbs plus chemotherapy, as did $44.7 \%(95 \% \mathrm{Cl}: 39.2 \%, 50.2 \%)$ of RAS wild-type patients with right-sided primary tumors.

- For RAS wild-type/BRAF mutant as well as RAS mutant/BRAF wild-type patients, the most common treatment was anti-VEGF mAbs plus chemotherapy with $44.4 \%(95 \% \mathrm{Cl}: 34.9 \%, 54.3 \%)$ and $61.1 \%(95 \% \mathrm{Cl}: 57.8 \%, 64.4 \%)$, respectively.

- There were differences between countries regarding the adopted treatment strategies with the UK generally prescribing less frequently anti-VEGF mAb plus chemotherapy compared with the other countries.

Supplementary data

To view the supplementary data that accompany this paper please visit the journal website at: www.futuremedicine.com/doi/suppl/10.2217/fon-2020-0976 
Financial \& competing interests disclosure

This research project was funded by Amgen Ltd. G Kafatos, D Neasham and KA Lowe are compensated employees of Amgen, Inc. and stockholders of Amgen, Inc. P Burdon was an employee of Amgen (Europe) GmbH at the time the research was conducted and owns shares in Amgen Inc.; he is currently an employee of MSD International GmbH. V Banks was a contract worker for Amgen, Ltd. at the time the research was conducted. C Anger and F Manuguid are compensated employees of IQVIA Ltd. J Trojan received consulting fees from Amgen, Bayer Healthcare, Bristol Myers-Squibb, Eisai, Ipsen, Merck Serono, Merck Sharp \& Dome, Lilly Imclone, Onkowissen TV, Pierre Fabre, PCI Biotech, Roche and Servier. He is serving on the speaker's bureau of Amgen, Bioprojet, Bristol Myers-Squibb, Eisai, Ipsen, Merck Serono, Merck Sharp \& Dome, Lilly Imclone, Roche, Servier and streammedup! and he received research grants from Roche and Ipsen. The authors have no other relevant affiliations or financial involvement with any organization or entity with a financial interest in or financial conflict with the subject matter or materials discussed in the manuscript apart from those disclosed.

The authors would like to thank and acknowledge M Hemetsberger of hemetsberger medical services, Vienna, Austria, for medical writing support, funded by Amgen.

Ethical conduct of research

The Oncology Dynamics database (IQVIA Ltd.) used in this study is fully anonymized and complies with relevant regulations for protecting patient privacy.

\section{Data sharing statement}

The data that support the findings of this study are available from IQVIA Ltd, but restrictions apply to the availability of these data, which were used under license for the current study, and so are not publicly available. Data are however available from the authors upon reasonable request and with permission of IQVIA Ltd.

\section{Open access}

This work is licensed under the Attribution-NonCommercial-NoDerivatives 4.0 Unported License. To view a copy of this license, visit http://creativecommons.org/licenses/by-nc-nd/4.0/

\section{References}

Papers of special note have been highlighted as: $\bullet$ of interest; $\bullet \bullet$ of considerable interest

1. Troiani T, Martinelli E, Morgillo F et al. Targeted approach to metastatic colorectal cancer: what comes beyond epidermal growth factor receptor antibodies and bevacizumab? Ther. Adv. Med. Oncol. 5(1), 51-72 (2013).

2. Ron DA, Vera R, Labandeira CM et al. Maintenance treatment in metastatic colorectal cancer: in search of the best strategy. Clin. Transl. Oncol. 22(8)1205-1215 (2020).

3. Moorcraft SY, Smyth EC, Cunningham D. The role of personalized medicine in metastatic colorectal cancer: an evolving landscape. Therap. Adv. Gastroenterol. 6(5), 381-395 (2013).

4. Boeckx N, Koukakis R, Op De Beeck Ket al. Primary tumor sidedness has an impact on prognosis and treatment outcome in metastatic colorectal cancer: results from two randomized first-line panitumumab studies. Ann. Oncol. 28(8), 1862-1868 (2017).

5. Lee GH, Malietzis G, Askari A, Bernardo D, Al-Hassi HO, Clark SK. Is right-sided colon cancer different to left-sided colorectal cancer? - A systematic review. Eur. J. Surg. Oncol. 41(3), 300-308 (2015).

6. Beets G, Sebag-Montefiore D, Andritsch E et al. ECCO essential requirements for quality cancer care: colorectal cancer. A critical review. Crit. Rev. Oncol. Hematol. 110, 81-93 (2017).

7. van Cutsem E, Cervantes A, Adam R et al. ESMO consensus guidelines for the management of patients with metastatic colorectal cancer. Ann. Oncol. 27(8), 1386-1422 (2016).

-. These are the current European Society for Medical Oncology guidelines for the management of metastatic colorectal cancer (mCRC) at the time of writing.

8. European Medicines Agency. Avastin summary of medicinal product characteristics. https://www.ema.europa.eu/en/documents/product-information/avastin-epar-product-information_en.pdf

9. European Medicines Agency. Vectibix summary of medicinal product characteristics. https://www.ema.europa.eu/en/documents/product-information/vectibix-epar-product-information_en.pdf

10. European Medicines Agency. Erbitux summary of medicinal product characteristics. https://www.ema.europa.eu/documents/product-information/erbitux-epar-product-information_en.pdf

11. Dankner M, Rose AN, Rajkumar S, Siegel PM, Watson IR. Classifying BRAF alterations in cancer: new rational therapeutic strategies for actionable mutations. Oncogene 37(24), 3183-3199 (2018). 
12. van Cutsem E, Huijberts S, Grothey A et al. Binimetinib, encorafenib, and cetuximab triplet therapy for patients with $B R A F$ v600e-mutant metastatic colorectal cancer: safety lead-in results from the Phase III BEACON colorectal cancer study. J. Clin. Oncol. 37(17), 1460-1469 (2019).

13. O’Dwyer PJ, Manola J, Valone FH et al. Fluorouracil modulation in colorectal cancer: lack of improvement with $\mathrm{N}$-phosphonoacetyl-1-aspartic acid or oral leucovorin or interferon, but enhanced therapeutic index with weekly 24-hour infusion schedule - an Eastern Cooperative Oncology Group/Cancer and Leukemia Group B Study. J. Clin. Oncol. 19(9), 2413-2421 (2001).

14. Arnold D, Lueza B, Douillard JY et al. Prognostic and predictive value of primary tumor side in patients with RAS wild-type metastatic colorectal cancer treated with chemotherapy and EGFR directed antibodies in six randomized trials. Ann. Oncol. 28(8), 1713-1729 (2017).

-. This meta-analysis was decisive in recognizing the prognostic and predictive potential of primary tumor location on the basis of six trials on EGFR-directed antibodies in combination with chemotherapy in $R A S$ wild-type mCRC.

15. Douillard JY, Oliner KS, Siena S et al. Panitumumab-FOLFOX4 treatment and RAS mutations in colorectal cancer. N. Engl. J. Med. 369(11), 1023-1034 (2013).

16. Peeters M, Price T, Taieb J et al. Relationships between tumor response and primary tumor location, and predictors of long-term survival, in patients with $R A S$ wild-type metastatic colorectal cancer receiving first-line panitumumab therapy: retrospective analyses of the PRIME and PEAK clinical trials. Br. J. Cancer 119(3), 303-312 (2018).

17. Schwartzberg LS, Rivera F, Karthaus $\mathrm{M}$ et al. PEAK: a randomized, multicenter phase II study of panitumumab plus modified fluorouracil, leucovorin, and oxaliplatin (mFOLFOX6) or bevacizumab plus mFOLFOX6 in patients with previously untreated, unresectable, wild-type KRAS exon 2 metastatic colorectal cancer. J. Clin. Oncol. 32(21), 2240-2247 (2014).

18. Tejpar S, Stintzing S, Ciardiello F et al. Prognostic and predictive relevance of primary tumor location in patients with $R A S$ wild-type metastatic colorectal cancer: retrospective analyses of the CRYSTAL and FIRE-3 trials. JAMA Oncol. 3(2), 194-201 (2017).

- Confirms the role of primary tumor location on the basis of cetuximab trials.

19. Schmidt N, Kostev K, Jockwig A, Kyvernitakis I, Albert US, Hadji P. Treatment persistence evaluation of tamoxifen and aromatase inhibitors in breast cancer patients in early and late stage disease. Int. J. Clin. Pharmacol. Ther. 52(11), 933-939 (2014).

20. Zhao Z, Pelletier E, Barber B et al. Major surgery in patients with metastatic colorectal cancer in Western Europe. J. Gastrointest. Cancer 43(3), 456-461 (2012).

21. Inoue Y, Toiyama Y, Tanaka K, Miki C, Kusunoki M. A comprehensive comparative study on the characteristics of colorectal cancer chemotherapy. Jpn. J. Clin. Oncol. 39(6), 367-375 (2009).

- This study used a predecessor of the current database to evaluate treatment patterns in the USA, the EU and Japan.

22. Karanis YB, Canta FaB, Mitrofan L, Mistry H, Anger C. 'Research' vs 'real world' patients: the representativeness of clinical trial participants. Ann. Oncol. 27(Suppl. 6), 526-544 (2016).

23. Canta FB, Mitrofan L, Karanis YB, Mistry H, Anger C. 17P deletion and TP53 gene mutation (17P/TP53) testing behaviour and treatment patterns for chronic lymphocytic leukemia (CLL) patients in France, Germany, Italy, Spain and UK (EU5). Ann. Oncol. 27(Suppl. 6), 313-327 (2016).

24. Cepparulo M, Schmidt N. Treatment of metastatic breast cancer in EU: analysis of market research data from 4Q2013 till 3Q2014. Breast 32, 256-265 (2017).

25. Marchetti P, Maass N, Gligorov J et al. Patient database analysis of fulvestrant $500 \mathrm{mg}$ in the treatment of metastatic breast cancer: A European perspective. Breast 32, 247-255 (2017).

26. Maroun R, Mitrofan L, Benjamin L et al. Real life patterns of care and progression free survival in metastatic renal cell carcinoma patients: retrospective analysis of cross-sectional data. BMC Cancer 18(1), 214 (2018).

27. Kafatos G, Banks V, Burdon P et al. Biomarker testing and mutation prevalence inmCRC patients in five European countries using a large oncology database. Future Oncol. doi:10.2217/fon-2020-0975 (2021) (Epub ahead of print).

28. Zhao Z, Pelletier E, Barber B et al. Patterns of treatment with chemotherapy and monoclonal antibodies for metastatic colorectal cancer in Western Europe. Curr. Med. Res. Opin. 28(2), 221-229 (2012).

- This study used a predecessor of the current database to evaluate treatment patterns in France, Germany, Italy and Spain.

29. Vectibix: procedural steps taken and scientific information after the authorisation. (2019). https://www.ema.europa.eu/en/documents/p rocedural-steps-after/vectibix-epar-procedural-steps-taken-scientific-information-after-authorisation_en.pdf

32. The National Institute for Health and Care Excellence (NICE). Bevacizumab in combination with oxaliplatin and either fluorouracil plus folinic acid or capecitabine for the treatment of metastatic colorectal cancer. http://www.nice.org.uk/guidance/ta212

33. The United Kingdom House of Commons Library. Removal of drugs from Cancer Drugs Fund list. http://researchbriefings.files.parliament.uk/documents/CDP-2016-0009/CDP-2016-0009.pdf

34. Zhou M, Yu P, Qu J et al. Efficacy of bevacizumab in the first-line treatment of patients with RAS mutations metastatic colorectal cancer: a systematic review and network meta-analysis. Cell Physiol. Biochem. 40(1-2), 361-369 (2016).

35. Midthun L, Shaheen S, Deisch J, Senthil M, Tsai J, Hsueh CT. Concomitant KRAS and BRAF mutations in colorectal cancer. J. Gastrointest. Oncol. 10(3), 577-581 (2019). 